\section{Interindividual variability in the expression of surfactant protein $A$ and $B$ in the human lung during development}

\author{
F. Cau, ${ }^{1}$ E. Pisu, ${ }^{2}$ C. Gerosa, ${ }^{3}$ G. Senes, ${ }^{3}$ \\ F. Ronchi, ${ }^{1}$ C. Botta, ${ }^{2}$ E. Di Felice, ${ }^{3}$ \\ F. Uda, ${ }^{3}$ V. Marinelli, ${ }^{4}$ G. Faa, ${ }^{3}$ V. Fanos, ${ }^{4}$ \\ C. Moretti, ${ }^{5}$ D. Fanni ${ }^{3}$ \\ 'Clinical Pathology, Hospital Nostra \\ Signora di Bonaria, ASL 6, San Gavino \\ 2Pathological Anatomy, Hospital Nostra \\ Signora di Bonaria, ASL 6, San Gavino \\ 3Pathological Anatomy, Hospital San \\ Giovanni di Dio, University of Cagliari \\ ${ }^{4}$ Neonatal Intensive Care Unit, Neonatal \\ Pathology, Puericulture Institute and \\ Neonatal Section, University of Cagliari \\ ${ }^{5}$ Department of Neonatology, Sapienza \\ University of Rome, Italy
}

\section{Abstract}

The surfactant complex, thanks to its multiple actions including decrease of surface-tension and antimicrobial activity, plays a fundamental role in newborn survival, lowering the risk of respiratory distress syndrome. The aim of this work was to determine if the synthesis of two surfactant proteins (SP), SPA and proSPB, shows some inter-individual variability during lung development in the intrauterine life. Immunoreactivity for SPA and pro-SPB was investigated in the lungs of 40 subjects, including 15 fetuses, ranging from 14 to 22 weeks of gestation, and 25 neonates, from 24 to 41 weeks. Lung samples were formalin fixed, paraffin-embedded and routinely processed. SPA and pro-SPB were detected utilizing commercial antibodies. A semi-quantitative grading system (1 to 4) was applied, based on the number of reactive cells and the intensity of immunostaining. Surfactant protein immunostaining was found in three compartments: bronchi, bronchioles and alveoli, starting from 14 weeks of gestation in the bronchial epithelium and from the $21^{\text {st }}$ week in the alveolar spaces. Differences were found regarding SPA and pro-SPB expression in the vast majority of subjects: in some lungs, SPA was more expressed whereas in others proSPB showed an higher degree of immunoreactivity. The expression of both surfactant proteins was not strictly correlated with gestational age. Whereas the highest levels of reactivity were detected in at term neonates, on the other hand one case with grade 3 was detected at 22 weeks and one negative case for both pro- teins was observed at 31 weeks. Our data clearly show a marked inter-individual variability regarding the production of SPA and pro-SPB and suggest the existence of other epigenetic factors, acting during gestation, that might influence surfactant production and, consequently, the survival potential of neonates at birth.

\section{Introduction}

Pulmonary surfactant is a lipoprotein complex composed of lipids (90\%) and proteins (10\%), secreted by the alveolar type II pneumocytes, that reduces the surface tension of fluids that coat alveoli, maintaining the stability of alveolar structures. Surfactant proteins include surfactant protein A (SPA), B (SPB), C (SPC), and D (SPD). ${ }^{1}$ Previous studies on SPA expression in the developing human fetal lung showed the first appearance of reactivity for SPA at 21 weeks of gestation in scattered epithelial cells in the main and segmental bronchi, whereas SPA immunostaining in alveolar type II cells appeared at 29 weeks, increasing until the $39^{\text {th }}$ week of gestation. ${ }^{2}$ The use of knockout mice evidenced the critical role of SPB in integrating the synthesis and metabolism of the surfactant complex, the absence of SPB being invariably fatal in the neonatal period. ${ }^{3}$ In other studies carried out in transgenic mice, the expression of mature SPB in type II cells was confirmed to be required for lung function, whereas SPB expression in club cells was not able to substitute this function. ${ }^{4}$ Further studies on SPB and SPC expression in the fetal human lung evidenced that both surfactant proteins are expressed primarily in distal bronchi and in terminal airway (bronchiolar) epithelial cells by the $15^{\text {th }}$ week of gestation, well in advance of expression in type II pneumocytes, starting around the $25^{\text {th }}$ week. ${ }^{5} \mathrm{~A}$ major role is played by SPB in the regulation of SPC synthesis, complete deficiency of SPB resulting in marked decreased levels of SPC. ${ }^{6}$ Recently, surfactant protein $\mathrm{C}$ has been reported in pulmonary mouse cells displaying proliferation, differentiation, and self-renewal capacity, suggesting the hypothesis that alveolar type II cells should be considered as progenitor cells for all alveolar epithelial cell types. ${ }^{7}$ These SPC-expressing bronchiolo-alveolar stem cells have been identified as alveolar epithelial progenitor cells, different in function from club cells that, according with this hypothesis, might represent the epithelial progenitors in trachea and bronchioles. ${ }^{8}$ The finding in the developing mouse of a preceding and overlapping expression of thyroid transcription factor-1 (TTF-1) in the same cells later expressing SPB and SPC supported the
Correspondence: Flaviana Cau, Patologia Clinica, Ospedale Nostra Signora di Bonaria, ASL 6, Via Roma, 09037 San Gavino (VS), Italy.

E-mail: flacau@tiscali.it

Key words: Surfactant; lung; immunohistochemistry; development.

Received for publication: 11 May 2016. Accepted for publication: 30 July 2016.

This work is licensed under a Creative Commons Attribution-NonCommercial 4.0 International License (CC BY-NC 4.0).

(O)Copyright F. Cau et al., 2016

Licensee PAGEPress, Italy

European Journal of Histochemistry 2016; 60:2678 doi:10.4081/ejh.2016.2678

hypothesis of a possible regulatory role for TTF-1 in lung expression of surfactant genes. ${ }^{9,10}$ A role for hepatocyte nuclear factor3beta (HNF3- $\beta$ ), a nuclear protein belonging to the winged family of transcription factors, has been assigned in the activation of multiple target genes critical for differentiation of respiratory epithelial cells, including TTF-1, $\mathrm{SPB}$, and Club cell secretory protein (CCSP). ${ }^{11}$ The earliest expression of SPA in the fetal human lung has been reported around the $15^{\text {th }}$ week of gestation, SPA-positive epithelial cells being mainly found in the larger bronchi. ${ }^{12} \mathrm{~A}$ delay in the synthesis of SPA by alveolar type II cells has been reported in the lung of newborns affected by congenital diaphragmatic hernia $(\mathrm{CDH})$, suggesting a role for SPA deficiency in the development of respiratory insufficiency often observed in these patients. ${ }^{13}$ SPD has been reported as the earliest surfactant protein to be expressed during lung development. SPD-reactivity has been reported in the bronchial epithelium since the $12^{\text {th }}$ week of gestation, gradually increasing during canalicular to saccular stage. During the saccular stage, immunoreactivity for SPD shifts from the bronchial epithelium to alveolar type II pneumocytes. ${ }^{14}$ In developing rat lung, the developmental profiles of SPA and SPB have been found to be different from each other: the former increased during late gestation, whereas SPB expression was low during gestation and increased after birth. ${ }^{15}$

On the basis of these data, it seemed of some interest to better analyze, by immunohistochemistry, SPA and pro-SPB expression during human development, in order to reach a better knowledge on the timing of expression of these surfactant proteins in bronchial, bronchiolar cells and in type II alveolar cells. In particular, this study was aimed at verifying if surfactant protein expression during human 
development shows any variability among different subjects, possibly being modulated by epigenetic factors acting during intrauterine life. Only SPA and SPB were evaluated because are generally considered to represent the most essential protein components of human lung surfactant.

\section{Patients and Methods}

This study was performed according the Code of Conduct of the Committee on Publication Ethics (COPE) and the COPE International Standards for Editors and Authors Guidelines. The human fetuses we received from the Obstetric Division of the University of Cagliari were as voluntary termination of pregnancy (VTOP) or from autopsy for diagnostic purpose. All the fetuses included in this study had no congenital malformation. All procedures performed were approved by the Ethics Human Studies Committee of University Medical Centre of Cagliari (according to the instructions of the Declaration of Helsinki).

This study was carried out on 40 subjects, including 15 fetuses, ranging from 14 to 22 weeks of gestation, and 25 neonates, from 24 to 41 weeks. Lung samples were formalinfixed by immersion in Neutral Buffered Formalin (NBF) for $24 \mathrm{~h}$, followed by processing in an Automatic processor for histology for $12 \mathrm{~h}$, then paraffin-embedded with a paraffin temperature closed to $50^{\circ} \mathrm{C}$ and routinely processed.

Serial sections $3 \mu \mathrm{m}$ thick were obtained from each paraffin block. Tissue sections were then dewaxed, rehydrated and pre-treated for immunohistochemical analysis with a $10-\mathrm{min}$ heat-induced epitope retrieval in buffer $\mathrm{pH}$ 6.00 (EnVisionTM FLEX Target Retrieval Solution Low pH - Dako Denmark A/S, Glostrup, Denmark; Code K8005). Slides were then incubated for $20 \mathrm{~min}$ at room temperature with anti-human Surfactant Protein A (SPA) mouse monoclonal antibody clone 32 E12 (NovocastraTM, Code NCL-SP-A) and with anti-human Pro-Surfactant Protein B (pro-SPB) mouse monoclonal antibody clone 19H7 (Abcam, Cambridge, UK; Catalog numbers ab49571), both at 1:100 dilution. Staining procedures were performed with the EnvisionTM FLEX+ (Dako, code K8002) Detection System and the AutostainerLink 48 instrument according to the manufacturer's instructions. A negative control, with the omission of the primary antibody, was performed (Supplementary Figure 1). Immunohistochemical staining for SPA and pro-SPB were carried out on all tissue lung included for each fetus. All tissue areas were used for the quantitative assessment and viewed for each fetus to directly compare the SPA and pro-SPB staining. Magnification for view of each compartment for the quantitative assessment were 40 and 63 HPF. A semi-quantitative grading system (1 to 4) was developed, based on the number of reactive cells and the intensity of immunostaining (Figure 1): grade 0, negative (Supplementary Figure 2); grade $1,<10 \%$ positive cells (Figure $1 \mathrm{~A}$ ); grade 2,10 $50 \%$ positive cells (Figure 1B); grade 3,51 $70 \%$ positive cells (Figure 1C); grade $4,>70 \%$ positive cells (Figure 1D). The counts are averages of all fields of lung view and evaluated. Surfactant protein immunostaining was evaluated in three compartments of the developing lungs: bronchi, bronchioles and alveoli. The association between the values of surfactant proteins and gestational age was assessed using Pearson's correlation coefficient (r). A P-value (P) $<0.05$ was considered statistically significant. All statistical analyses were performed using the statistical package for social science (SPSS) software. In same cases the clinician administrated surfactant: case 19 at 25 weeks, case 24 at 28 weeks, cases $26,27,28$ at 30 weeks, case 33 at 36 weeks, and case 36 at 39 weeks.

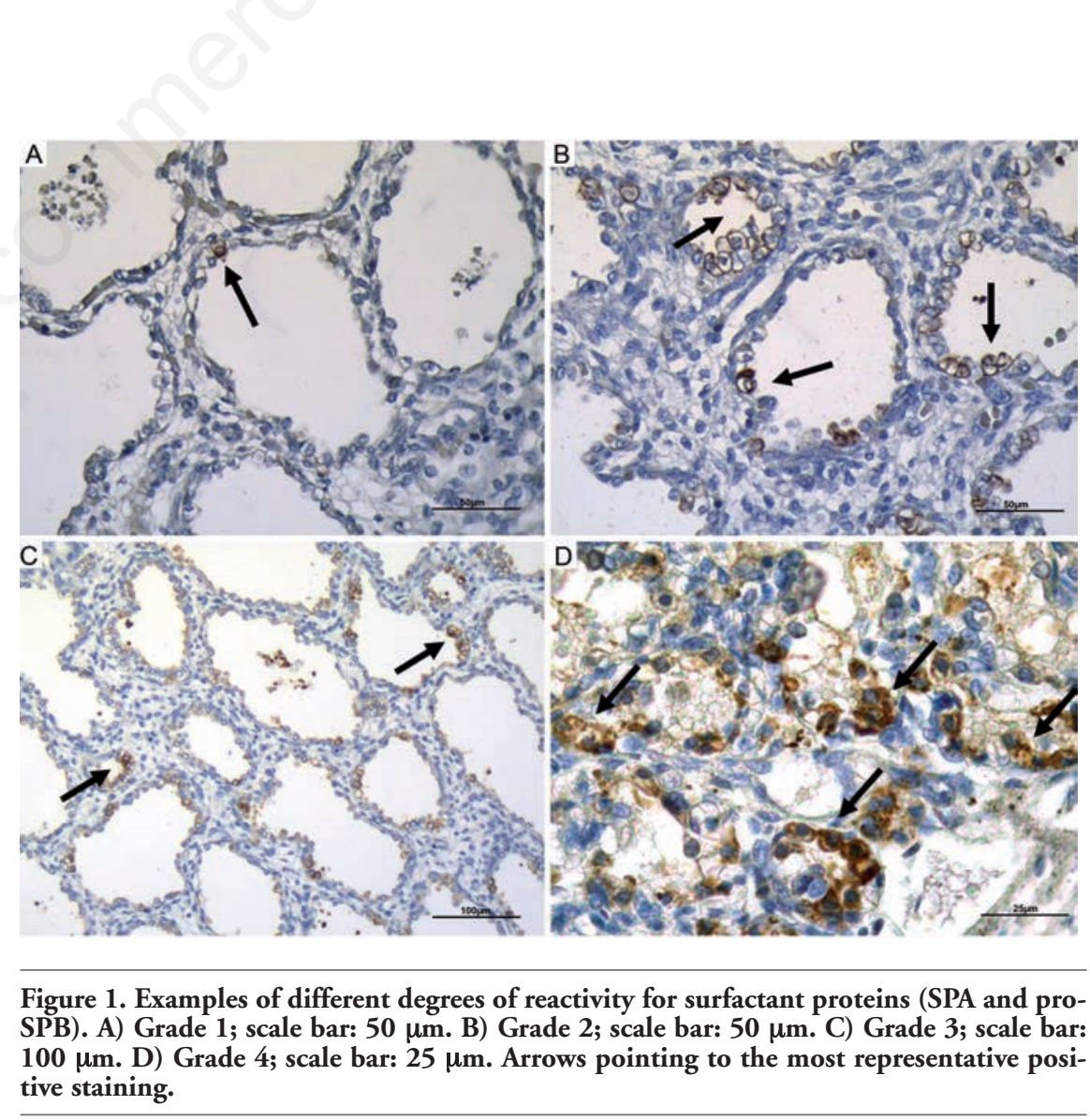

Figure 1. Examples of different degrees of reactivity for surfactant proteins (SPA and proSPB). A) Grade 1; scale bar: $50 \mu \mathrm{m}$. B) Grade 2; scale bar: $50 \mu \mathrm{m}$. C) Grade 3; scale bar: $100 \mu \mathrm{m}$. D) Grade 4; scale bar: $25 \mu \mathrm{m}$. Arrows pointing to the most representative positive staining.

\section{Results}

Both surfactant proteins SPA and pro-SPB showed a preferential cytoplasmic pattern staining and both were observed in three compartments of the developing lung: the bronchial epithelium, the bronchiolar epithelium and the alveolar epithelium. In some cases, in addition, reactivity for surfactant proteins was also found in the alveolar spaces and in the bronchiolar lumen. The expression of SPA showed no marked differences when compared with pro-SPB expression. For this reason, the results of the two surfactant proteins will be reported separately.

\section{SPA}

i) Bronchial reactivity. Immunostaining for SPA was detected in 18 out of 40 (45\%) lungs in the bronchial epithelium (Table 1). This surfactant protein was expressed in the cytoplasm of scattered epithelial cells that, at morphology, did not differ from the neighboring bronchial epithelial cells (Figure 2 A-D). Even though the three cases showing the highest degree of bronchial reactivity for SPA (grade 3 and 4) were all at term, on the other hand the expression of this surfactant protein was not strictly related to the gestational age of fetuses 
and newborns. SPA was expressed in two 14-week-old fetuses, but it was not detected in four neonates of 30 weeks. Differences were also found among fetuses of the same gestational age: at 21 weeks, one showed grade 2 , another grade 1 positivity, whereas in the other three 21 week-old subjects SPA was not found (Table 1).

ii) Bronchiolar reactivity. Immunoreactivity for SPA was observed in 21 cases (52\%) in the bronchiolar epithelium, SPA being detected in the cytoplasm of scattered cells intermingled between the epithelium covering the small airways (Figure $2 \mathrm{E}-\mathrm{H}$ ). The expression of SPA in the bronchioles was not strictly related to the gestational age: in particular, fetuses (see 21 weeks) and neonates (see 30 weeks, Table 1) of the same gestational age showed significant differences among them regarding the expression or not of the protein in the bronchiolar lining cells. The highest levels (grade 4) of immunostaining for bronchiolar SPA have been observed in infants born at $39-40$ weeks of gestation (Table 1).

iii) The alveolar expression of SPA appeared later: the first mild reactivity on the alveolar epithelium was detected in three fetuses of 21 weeks of gestation, other two fetuses carrying the same gestational age being negative at alveolar level (Table 1). The expression of SPA in the alveolar epithelial cells was not related to gestational age: grade 3 of immunostaining was found at $22,33,36$ and 40 weeks of gestation. Changes were also found in newborns of the same gestational age: in 39 week-old neonates, alveolar reactivity for SPA ranged from grade 1 to grade 4 , whereas a newborn of 41 weeks showed grade 2 immunostaining (Table 1; Figure 2 I-L).

\section{Pro-SPB}

i) Bronchial reactivity. This surfactant protein was found, at bronchial level, in 20 out of 40 cases analyzed (50\%) (Table 1). Pro-SPB protein was expressed in the cytoplasm of scattered cells intermingled between the bronchial epithelium (Figure 3 A-D). Pro-SPB expression did not correlate with gestational age. Moreover, the highest levels of pro-SPB bronchial expression (grade 3 ) were observed at different gestational ages, ranging from 21 up to 40 weeks (Table 1).

ii) Bronchiolar reactivity. In the bronchiolar epithelium, scattered pro-SPB-reactive cells were found in 20 out of 40 cases (50\%) (Figure $3 \mathrm{E}-\mathrm{H})$. pro-SPB expression in the small airways was first found in two 14 week-old fetuses. It was not correlated with the gestational age and was frequently different even in fetuses and newborns of the same age. In 30 weekold newborns, the degree of pro-SPB immunoreactivity ranged from 0 to 2 (Table 1 ).

iii) Alveolar reactivity. The expression of

Table 1. SPA and pro-SPB expressions in bronchi, bronchioles and alveoli at different gestational ages.

\begin{tabular}{|c|c|c|}
\hline Subjects & $\begin{array}{l}\text { Surfactant } \\
\text { therany }\end{array}$ & $\begin{array}{l}\text { Gestational age } \\
\text { (weeks) }\end{array}$ \\
\hline
\end{tabular}

\begin{tabular}{llllllll}
1 & 14 & 1 & 0 & 0 & 2 & 2 & 0 \\
2 & 14 & 2 & 2 & 0 & 2 & 2 & 0 \\
\hline 3 & 16 & 0 & 0 & 0 & 0 & 0 & 0 \\
4 & 17 & 1 & 1 & 0 & 1 & 0 & 0 \\
\hline 5 & 17 & 1 & 0 & 0 & 1 & 1 & 0
\end{tabular}

\begin{tabular}{llllllll}
6 & 18 & 0 & 0 & 0 & 0 & 0 & 0 \\
\hline 7 & 19 & 0 & 0 & 0 & 0 & 0 & 0
\end{tabular}

\begin{tabular}{llllllll}
8 & 21 & 0 & 0 & 0 & 0 & 0 & 0 \\
\hline 9 & 21 & 0 & 0 & 0 & 0 & 0 & 0
\end{tabular}

\begin{tabular}{lllllllll}
10 & 21 & 0 & 1 & 1 & 0 & 1 & 2 \\
\hline 11 & 21 & 2 & 1 & 1 & 1 & 1 & 0
\end{tabular}

$\begin{array}{llllllll}12 & 21 & 1 & 1 & 1 & 3 & 2 & 2 \\ 13 & 22 & 0 & 0 & 0 & 1 & 0 & 0\end{array}$

\begin{tabular}{llllllll}
14 & 22 & 1 & 1 & 1 & 1 & 1 & 1 \\
\hline 15 & 22 & 1 & 0 & 3 & 2 & 2 & 1
\end{tabular}

$\begin{array}{llllllll}16 & 24 & 2 & 1 & 0 & 1 & 0 & 0 \\ 17 & 24 & 0 & 0 & 0 & 0 & 0 & 0\end{array}$

\begin{tabular}{lllllllll}
18 & 25 & 1 & 1 & 1 & 3 & 2 & 2 \\
\hline 19 & S+ & 25 & 1 & 2 & 2 & 2 & 2 & 3
\end{tabular}

\begin{tabular}{llllllll}
20 & 26 & 0 & 0 & 0 & 0 & 0 & 0 \\
\hline 21 & 26 & 1 & 1 & 1 & 2 & 2 & 0 \\
22 & 27 & 0 & 0 & 0 & 0 & 0 & 0 \\
23 & 28 & 0 & 0 & 0 & 0 & 0 & 0
\end{tabular}

\begin{tabular}{lllllllll}
24 & $\mathrm{~S}+$ & 28 & 0 & 0 & 0 & 0 & 0 & 0 \\
\hline 25 & & 29 & 0 & 1 & 1 & 0 & 1 & 0
\end{tabular}

\begin{tabular}{lllllllll}
26 & $S+$ & 30 & 1 & 1 & 1 & 1 & 0 & 0 \\
\hline 27 & $S+$ & 30 & 0 & 0 & 0 & 0 & 0 & 0 \\
28 & $S+$ & 30 & 0 & 1 & 0 & 0 & 2 & 3 \\
\hline 29 & & 30 & 0 & 2 & 0 & 0 & 1 & 3 \\
30 & 30 & 0 & 1 & 1 & 0 & 0 & 0 \\
\hline 31 & 31 & 0 & 0 & 0 & 0 & 0 & 0 \\
32 & & 33 & 0 & 0 & 3 & 0 & 0 & 0 \\
33 & & 36 & 1 & 3 & 3 & 2 & 3 & 2 \\
34 & & 38 & 0 & 0 & 1 & 0 & 0 & 0 \\
35 & & 39 & 1 & 2 & 1 & 2 & 2 & 2 \\
36 & & 39 & 4 & 4 & 4 & 3 & 3 & 3 \\
37 & $5+$ & 40 & 0 & 1 & 3 & 0 & 1 & 0 \\
38 & & 40 & 4 & 4 & 4 & 1 & 1 & 1 \\
\hline 39 & & 40 & 3 & 4 & 4 & 3 & 2 & 1 \\
40 & & 41 & 0 & 0 & 2 & 1 & 0 & 1 \\
\hline
\end{tabular}


pro-SPB in the alveolar epithelium was first observed at 21 weeks of gestation. Immunoistaining for pro-SPB appeared as a thin reactive layer covering the surface of the alveolar epithelium (Figure $3 \mathrm{I}-\mathrm{L}$ ). No correlation ( $\mathrm{P}>0.05$ ) was found between pro-SPB alveolar reactivity and gestational age. The highest degree (grade 3) of pro-SPB immunostaining was detected at different gestational ages, ranging from 25 up to 39 weeks of gestation (Table 1).

When SPA and pro-SPB expression were compared, marked differences were detected between their expression in all three lung compartments examined. At bronchial level (Figure 4), pro-SPB showed a higher degree of reactivity in 11 cases, whereas SPA was more expressed in 6 cases. In some subjects, the two surfactant proteins showed striking differences regarding their expression: in a 40 week-old newborn, the low degree of reactivity for pro-SPB grade 1 contrasted with grade 4 for SPA. Marked differences between the two surfactant proteins in the same lung were also observed in the small airways: a predominant expression of SPA on pro-SPB, grade 4 to 1 and grade 4 to 2, was observed in bronchioles of two at term newborns of 40 weeks, whereas the other 40 week-old neonates showed a equal expression of pro-SPB at bronchiolar level (Figure 5). The inter-individual variability in surfactant protein expression at bronchiolar level is well evidenced by two preterms of 36 and 38 weeks of gestation: in the younger, SPA and pro-SPB were highly expressed (grade 3 ); in the 38-old newborn, an opposite pattern was present, characterized by lack of expression of pro-SPB and SPA (Figure 5).

Different patterns of expression were also found regarding the expression of SPA and pro-SPB at alveolar level (Figure 6). Whereas the highest values of SPA expression (grade 4) were concentrated in the last weeks of gestation, pro-SPB expression appeared more irregular, its highest values (grade 3 ) being detected at different gestational ages, ranging from 25 to 39 weeks of gestation (Figure 6). The marked interindividual variability in the expression of these two surfactant proteins is well evidenced by the analysis of the five 30 week-old neonates: one was devoid of both proteins; two showed grade 1 reactivity for SPA and absence of pro-SPB; the other two were characterized by a marked expression of proSPB (grade 3) in the absence of any reactivity for SPA (Figure 6). The other newborn carrying the same gestational age (30 weeks), showed a completely opposite pattern: the strong expression of pro-SPB (grade 3) contrasted with the absence of any alveolar reactivity for SPA.

Statistical analyses evidenced a significant and positive correlation $(\mathrm{r}=0.65 ; \mathrm{P}<0.01)$

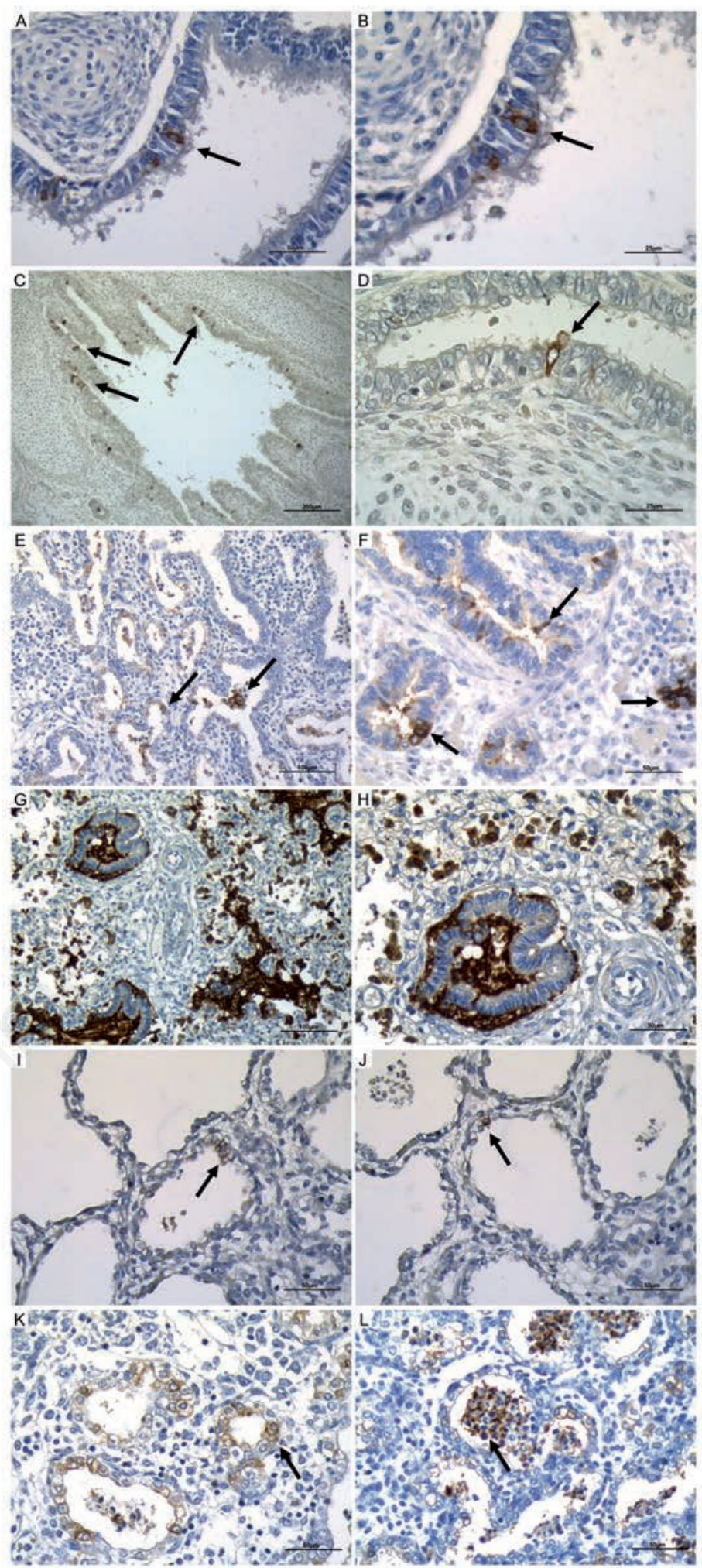

Figure 2. Immunohistochemical expression of SPA in bronchial epithelial cells, in bronchiolar epithelial cells and in alveolar epithelium at different magnification. A) Bronchial epithelial cells; scale bar: $50 \mu \mathrm{m}$. B) Bronchial epithelial cells; scale bar: $25 \mu \mathrm{m}$. C) Bronchial epithelial cells; scale bar: $200 \mu \mathrm{m}$. D) Bronchial epithelial cells; scale bar: 25 $\mu \mathrm{m}$. E) Bronchiolar epithelial cells; scale bar: $100 \mu \mathrm{m}$. F) Bronchiolar epithelial cells; scale bar: $50 \mu \mathrm{m}$. G) Bronchiolar epithelial cells scale; bar: $100 \mu \mathrm{m}$. H) Bronchiolar epithelial cells; scale bar: $50 \mu \mathrm{m})$; I,J) Alveolar epithelium at 25 weeks of gestational age with different alveolar dimension; scale bar: $50 \mu \mathrm{m}$. K,L) Alveolar epithelium at 22 weeks of gestational age with different alveolar shape and branching; scale bar: $50 \mu \mathrm{m}$. Arrows pointing to the most representative positive staining. 
between the expression of alveolar SPA and gestational age. In fact, in spite of the interindividual variability at all gestational ages, the highest values (3 and 4) are more frequent in the last months of gestation, starting from the $31^{\text {st }}$ week, whereas 0 values are more concentrated in the first weeks (Figure 7). The association between SPA expression and gestational age $(\mathrm{r}=0.63 ; \mathrm{P}<0.01)$ was confirmed when subjects were subdivided into three groups according with the different gestational age: early (14-21 weeks), intermediate (22-30) and late (31-41); 75\% of fetuses included in the early group did not show any reactivity for SPA; the percentage of non reactive cases was $55 \%$ in the intermediate group and $10 \%$ in the late group (Figure 7 ).

With regard to SPA values in bronchi and bronchioles, and pro-SPB values in all pulmonary compartments of fetuses and newborns, statistic analyses confirmed the absence of any significant correlation with gestational age.

\section{Discussion}

The surfactant complex plays multiple relevant roles in the early phases of human development, protecting the fetus during gestation and the newborn at birth. The multiple surfactant components decrease alveolar surface tension, bind and inactivate bacteria and viruses, facilitate their phagocytosis by alveolar macrophages and modulate inflammation by decreasing harmful inflammatory responses. ${ }^{16}$ As a consequence, surfactant deficiency at birth, due to lack of mature alveolar type II epithelial cells, is associated with the development of respiratory distress syndrome (RDS) and hyaline membrane disease. ${ }^{17}$ In recent years, surfactant dysfunction due to multiple mechanisms including protein leakage into alveolar spaces $^{18}$ and meconium inhalation ${ }^{19}$ has been associated with the insurgence of acute respiratory distress syndrome (ARDS) in pediatric patients affected by pneumonia and severe bronchiolitis. ${ }^{20}$ Given the relevant role of a proper surfactant synthesis and secretion in the optimal development of the human lung, this study was aimed at improving the knowledge on the timing of the production of two main surfactant components, SPA and pro$\mathrm{SPB}$, during gestation.

Our data first evidence a marked interindividual variability in the expression of both SPA and pro-SPB among the 40 fetuses and newborns analyzed. This variability regards surfactant protein expression in three pulmonary compartments, including bronchial, bronchiolar and alveolar epithelium. High degrees of SPA and pro-SPB bronchial and/or alveolar

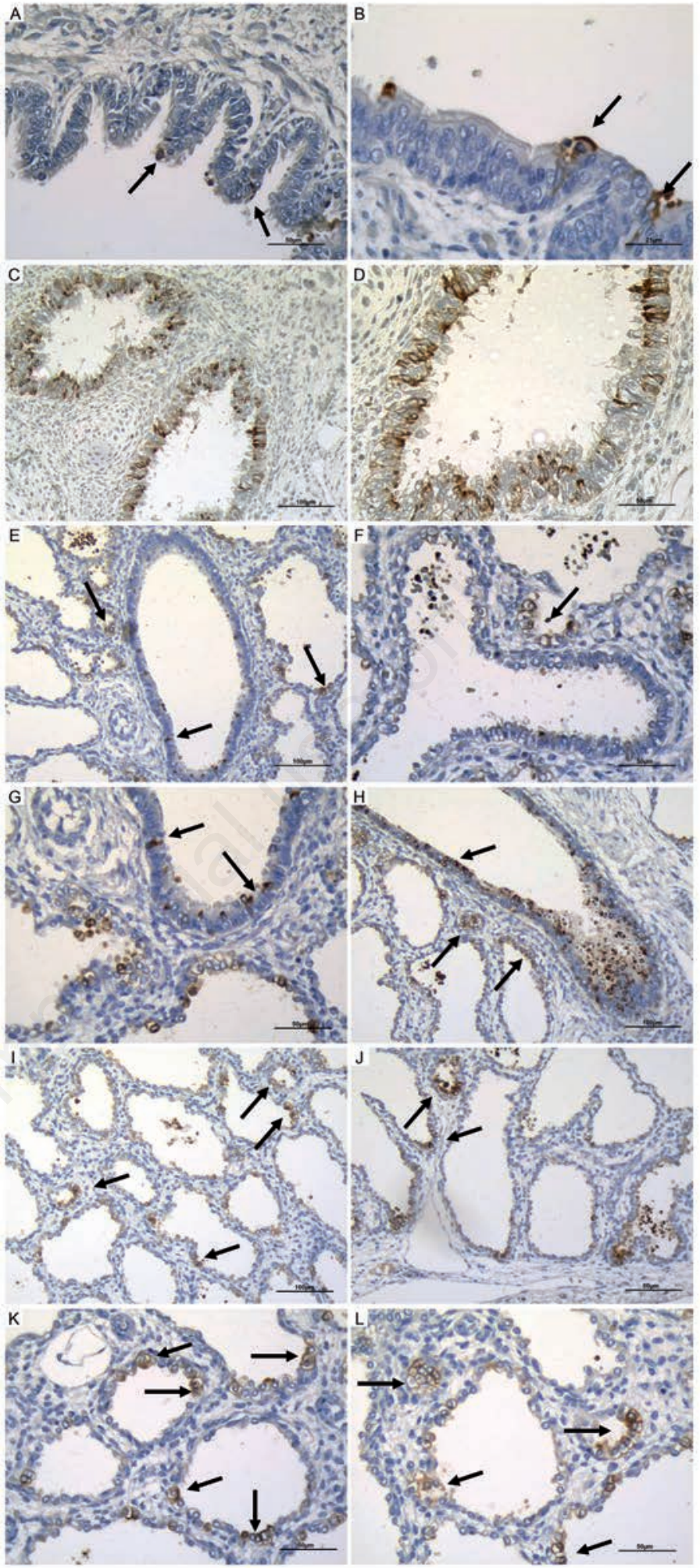

Figure 3. Pro-SPB immunoreactivity in bronchial epithelial cells, in bronchiolar epithelial cells and in alveolar epithelium at different magnification. A) Bronchial epithelial cells; scale bar: $50 \mu \mathrm{m}$. B) Bronchial epithelial cells; scale bar: $25 \mu \mathrm{m}$. C) Bronchial epithelial cells; scale bar: $100 \mu \mathrm{m}$. D) Bronchial epithelial cells; scale bar: $50 \mu \mathrm{m}$. E) Bronchial epithelial cells; scale bar: $100 \mu \mathrm{m}$. F) Bronchiolar epithelial cells; scale bar: $\mathbf{5 0}$ $\mu \mathrm{m}$. G) Bronchiolar epithelial cells; scale bar: $50 \mu \mathrm{m}$. H) Bronchiolar epithelial cells; scale bar: $100 \mu \mathrm{m}$. I) Alveolar epithelium; scale bar: $100 \mu \mathrm{m}$. J) Alveolar epithelium at 25 weeks of gestational age with different alveolar dimension; scale bar: $50 \mu \mathrm{m}$. K, L) Alveolar epithelium with different alveolar shape; scale bar: $50 \mu \mathrm{m}$. Arrows pointing to the most representative positive staining. 
expression were found in some fetuses at very early gestational ages (21 weeks), suggesting their definition as fast surfactant producers; on the other extreme of the spectrum, the absence or a very low level of expression was detected in at term babies, indicating the existence of late surfactant producers. Marked interindividual variability was observed in the cases with surfactant administration as well, even at the same gestational age, for example cases 26,27 and 30 , all at 30 weeks, showed too many differences in the grading of the expression either of SPA and pro-SPB. In fact this 7 cases were not enough to allow us any speculation of the specific therapy.

Statistical analyses evidenced a significant correlation between surfactant expression and gestational age restricted to SPA alveolar secretion. No correlation with gestational age was found between SPA bronchial and bronchiolar expression and pro-SPB expression. These data taken together indicate that other genetic or epigenetic factors may influence significantly surfactant protein production, leading to marked differences in SPA and proSPB expression in the newborn lungs. Regarding the timing of surfactant protein production during the intrauterine life, in this study both SPA and pro-SPB were first detected in bronchial and bronchiolar epithelium at 14 weeks of gestation, confirming previous data from Xu et al. ${ }^{12}$ The first expression at alveolar level of both SPA and pro-SPB was observed starting from the $21^{\text {st }}$ week of gestation: this finding suggests, a more precocious production than previously reported by Endo et al. ${ }^{2}$ and by Khoor et al..$^{21}$ The synthesis of SPA and pro-SPB appears, on the basis of our data, completely independent. Only in few cases, the degree of immunoreactivity was the same. In the vast majority of cases, marked differences were found regarding SPA and pro-SPB production. Moreover, given the major role played by pro-SPB in lung function in the perinatal period, ${ }^{6}$ an interesting datum emerging by our study is the detection of low pro-SPB levels in the majority of newborns with more than 29 weeks of gestation. Among 15 neonates aging 30-41 weeks, seven did not show any reactivity (grade 0), three showed grade 1, two had grade 2, three had grade 3 , and none showed grade 4 reactivity. These findings may suggest a prevalent postnatal production of pro-SPB, at least in the majority of neonates.

In conclusion, our data clearly indicate that the production of two components of the human surfactant complex, SPA and pro-SPB is characterized by a marked interindividual variability. These findings suggest that a reduced surfactant synthesis may occur during gestation, probably due to a delay in maturation or to injury of type II pneomocytes and/or bronchial and bronchiolar epithelial cells.

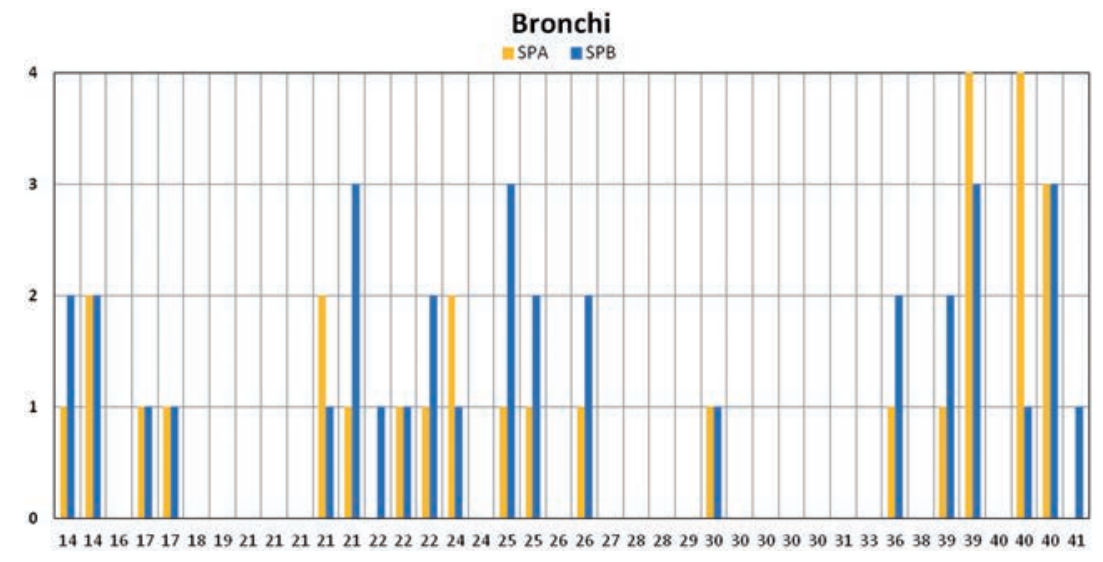

Figure 4. Histogram graphical representation of SPA and pro-SPB expression in bronchi at different gestational age showind the higher degree of reactivity of pro-SPB in 11 cases, SPA in 6 cases and the differences between the two surfactant proteins.

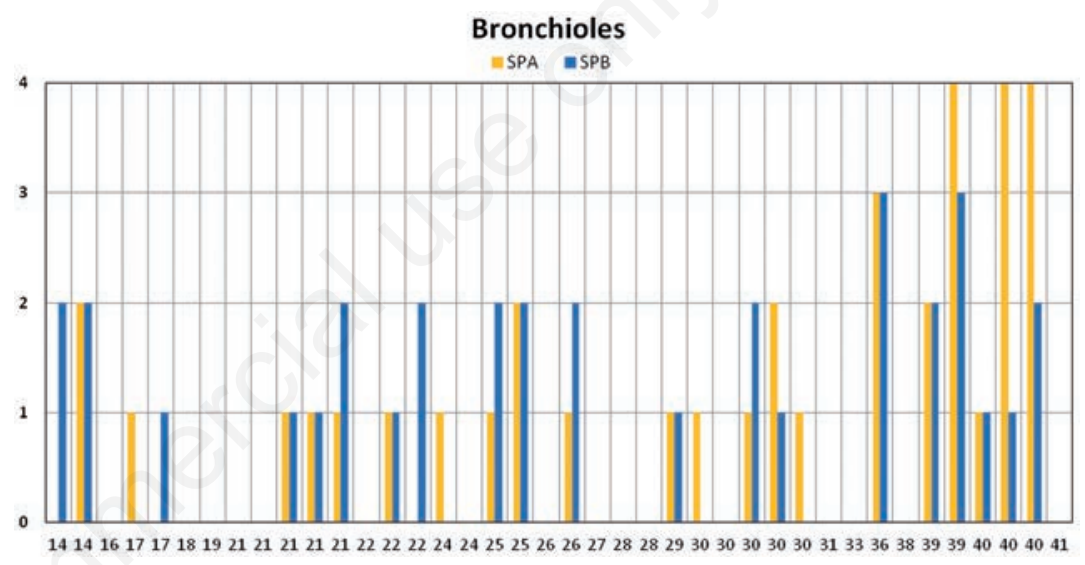

Figure 5. Histogram graphical representation of SPA and pro-SPB expression in bronchioles at different gestational age showing the marked differences between SPA and proSPB in the same lung were also observed in the small airways and the inter-individual variability.

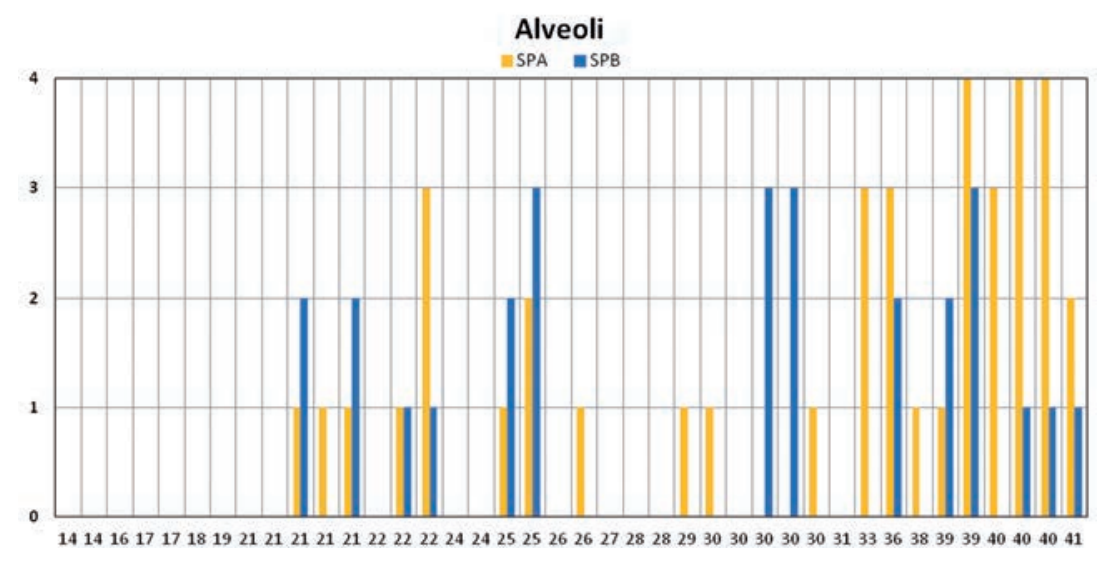

Figure 6. Histogram graphical representation of SPA and pro-SPB expression in alveolar epithelium at different gestational age with the highest values of SPA expression in the last weeks of gestation and showing the irregular pro-SPB expression with the highest values from 25 to 39 weeks of gestation. 


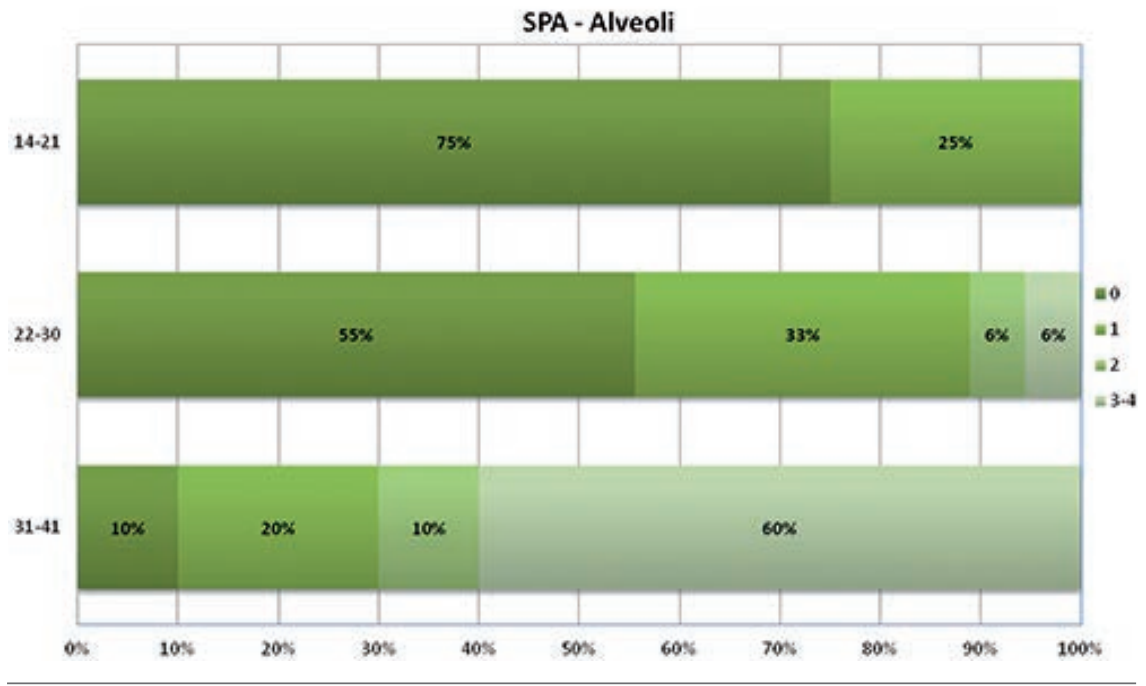

Figure 7. Bar graphical representation showing the percentage of SPA immunohistochemical expression into three groups of gestational age: 14-21 weeks, 22-30 weeks and 31-41 weeks. The highest reactivity were observed from the $31^{\text {st }}$ week.

Further studies should be aimed at analyzing the pathways by which type II cells might be injured during gestation, ending with a decrease or with the absence of one or multiple surfactant components at birth.

\section{References}

1. Goerke J. Pulmonary surfactant: functions and molecular composition. Biochim Biophys Acta 1998;1408:79-89.

2. Endo H, Oka T. An immunohistochemical study of bronchial cells producing surfactant protein $\mathrm{A}$ in the developing human fetal lung. Early Hum Dev 1991;25:149-56.

3. Weaver TE, Beck DC. Use of knockout mice to study surfactant protein structure and function. Biol Neonate 1999;76:15-8.

4. Lin S, Na CL, Akinbi HT, Apsley KS, Whitsett JA, Weaver TE. Surfactant protein B (SP-B)-/- mice are rescued by restoration of SP-B expression in alveolar type II cells but not Clara cells. J Biol Chem 1999;274:19168-74.

5. Khoor A, Stahlman MT, Gray ME, Whitsett J. Temporal-spatial distribution of SP-B and SP-C proteins and mRNAs in developing respiratory epithelium of human lung.
J Histochem Cytochem 1994;42:1187-99.

6. Weaver TE, Conkright JJ. Function of surfactant proteins B and C. Annu Rev Physiol 2001;63:555-78.

7. Lee J-H, Kim J, Gludish D, Roach RR, Saunders AH, Barrios J, et al. Surfactant Protein-C Chromatin-Bound Green Fluorescence Protein Reporter Mice Reveal Heterogeneity of Surfactant Protein C-Expressing Lung Cells. Am J Respir Cell Mol Bio 2013;48:288-98.

8. Rawlins EL, Okubo T, Xue Y, Brass DM, Auten RL, Hasegawa H, et al. The role of Scgbla1+ Clara cells in the long-term maintenance and repair of lung airway, but not alveolar, epithelium. Cell Stem Cell 2009;4:525-34.

9. Zhou L, Lim L, Costa RH, Whitsett JA. Thyroid transcription factor-1, hepatocyte nuclear factor-3beta, surfactant protein B, $\mathrm{C}$, and Clara cell secretory protein in developing mouse lung. J Histochem Cytochem 1996;44:1183-93.

10. Cao Y, Vo T, Millien G, Tagne J-B, Kotton D, Mason RJ, et al. Epigenetic mechanisms modulate thyroid transcription factor 1mediated transcription of the surfactant protein B gene. J Biol Chem 2010;285: 2152-64.

11. Zhou L, Dey CR, Wert SE, Yan C, Costa RH,
Whitsett JA. Hepatocyte nuclear factor $3 \beta$ limits cellular diversity in the developing respiratory epithelium and alters lung morphogenesis in vivo. Dev Dyn 1997;210:305-14.

12. Xu P, Hashimoto S, Miyazaki H, Asabe K, Shiraishi S, Sueishi K. Morphometric analysis of the immunohistochemical expression of Clara cell $10-\mathrm{kDa}$ protein and surfactant apoproteins A and B in the developing bronchi and bronchioles of human fetuses and neonates. Virchows Arch 1998;432:17-25.

13. Asabe K, Tsuji K, Handa N, Kurosaka N, Kajiwara M. Immunohistochemical distribution of surfactant apoprotein-A in congenital diaphragmatic hernia. J Pediatr Surg 1997;32:667-72.

14. Chen Y, Du J, Zhao Q, Feng Z. [Expression of pulmonary surfactant-associated protein D and its significance in human fetal lung].[Article in Chinese]. Di Yi Jun Yi Da Xue Xue Bao 2005;25:329-31.

15. Shimizu H, Miyamura K, Kuroki Y. Appearance of surfactant proteins, SP-A and SP-B, in developing rat lung and the effects of in vivo dexamethasone treatment. Biochim Biophys Acta 1991;1081:5360 .

16. Hallman M. The surfactant system protects both fetus and newborn. Neonatology 2013;103:320-6.

17. Locci G, Fanos V, Gerosa C, Faa G. Hyaline membrane disease (HMD): the role of the perinatal pathologist. JPNIM 2014;3: $\mathrm{e} 030255$.

18. Seeger W, Stohr G, Wolf H, Neuhof H. Alteration of surfactant function due to protein leakage: special interaction with fibrin monomer. J Appl Physiol 1985;58:326-38.

19. Moses D, Holm BA, Spitale P, Liu M, Enhorning G. Inhibition of pulmonary surfactant function by meconium. Am J Obstet Gynecol 1991;164:477-81.

20. Moretti C, Barbàra CS, Grossi R, Luciani S, Midulla F, Papoff P. Surfactant therapy for acute respiratory distress in infants. JPNIM 2014;3:e030238.

21. Khoor A, Stahlman MT, Gray ME, Whitsett JA. Temporal-spatial distribution of SP-B and SP-C proteins and RNAs in developing respiratory epithelium of human lung. $\mathrm{J}$ Histochem Cytochem 1994;42:1187-99. 